\title{
AUTOMATED SYSTEM FOR REMOTE DEFECT INSPECTION
}

\author{
Lopot František ${ }^{1}$, Daniel Hadraba ${ }^{2}$, Petr Kubový3, Jan Hošek ${ }^{4}$ \\ 1,2,4 Faculty of Mechanical Engineering, Czech Technical University in Prague \\ Technicka 4, Prague 6, Czech Republic \\ ${ }^{3}$ Faculty of Physical Education and Sport, Charles University \\ José Martího 31, Prague 6, Czech Republic \\ flopot@seznam.cz,daniel.hadraba@fs.cvut.cz,jan.hosek@fs.cvut.cz
}

\begin{abstract}
The paper deals with development and application of a method which allows to perform automatic check the surface integrity of the monitored component. In our case, it is the bearing race of the rotating cylindrical container of the lime regeneration line in the paper mill factory. By visual checks, some cracks have been discovered on the circular surface of the race. These cracks have needed to be monitored to prevent fatal failure. To measure the cracks manually, it was always necessary to stop the cylinder. However, these breaks have caused momentous problems in the operation of the line, which is conceived for continuous work due to the nature of the production process. This was the main reason for the development and deployment of the automated system. Because the problem takes of low-frequency bearing run (less than $1 \mathrm{~Hz}$ ) moreover used in outdoor conditions an image processing technology was chosen to create a tracking system to determine the immediate crack length at specified times.

Due to the very limited time span for the development of a separate robust apparatus and with regards to the fact that the system is used only till replacing the concerned race with a new one, i.e. for a period of about three months, the hardware of the apparatus was based on a standard web camera with fullHD resolution connected to a notebook with a controlling and recording software created in the Matlab environment. This "recording" system located close to the monitored bearing is wirelessly connected to the "controlling" computer placed in the control center of the paper mill. The system reduces the data stream of optical scanning of $750 \mathrm{~mm}$ wide bearing ring by cut of the recorded figures to a data level that reliably enables their further processing and evaluation. It is done by the control center computer.

The system is able to indicate individual cracks present at the bearing ring surface with image data processing. Documentation and assessment of development of the dimensions of identified and observed cracks are also presented.

For a better idea about all aspects of the problem, we add that the ring has the diameter of $4.7 \mathrm{~m}$ and scanning width of almost $800 \mathrm{~mm}$ due to additional axial motion.
\end{abstract}

Keywords: Michelson interferometer, developed three-longitudinal mode heterodyne interferometer, nonlinearity, nonlinearity compensation.

\section{Introduction}

The collaborating paper mill factory works with very modern equipment, which allows, but at the same time supposes, non-stop operation. Any technical problems in virtually any part of the line cause irreversible degradation of raw materials in a very short time. Another problem is the fact that the stopping and start-up of some parts of the production lines, which simultaneously act as raw material trays, is a relatively long-term and technically demanding process which further increases the reported losses. Also one cannot ignore possible ecological risks.

An example of such a machine is the line for lime regeneration by a hydroxide. It is based on a mixing roller with a diameter of almost $4 \mathrm{~m}$ with a total length of about $40 \mathrm{~m}$ with rake angle of about $4^{\circ}$ against the horizontal, which is filled up to the half of its radius at the highest point (Fig. 1).

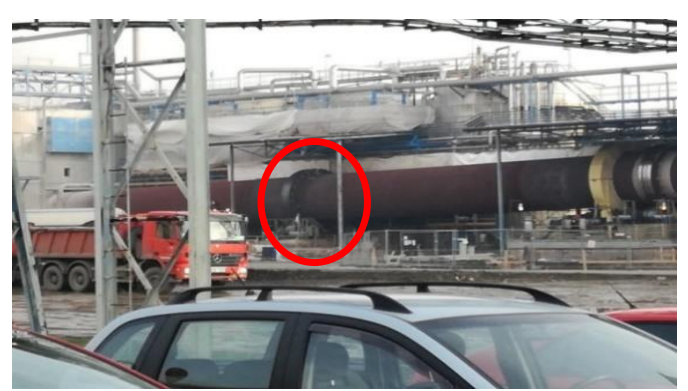

Figure 1: View on mixing cylinder and examined support (marked by red ellipse) 
The cylinder is located in an open space with partially covered by metal sheet roofs. It is placed on several support rings, which rotate with it on two support rollers. The contact surfaces are lubricated at prescribed intervals and, due to the lubrication process, the cylinder is moved in the axial direction by +/- $150 \mathrm{~mm}$ around the nominal position. One revolution of the cylinder during normal line operation takes approx. $55 \mathrm{~s}$. This time extends up to 2.5 minutes whenever the regular maintenance lubrication is performed. In terms of installation of the measuring apparatus, also the temperature is important - it exceeds $80^{\circ} \mathrm{C}$ at the surface of the cylinder. The surface of the support ring has a temperature of about $35^{\circ} \mathrm{C}$. This difference is achieved by the design of the support ring which is conceived as a hollow with go-through holes for free air flow which effectively removes the heat emitted by the mixing cylinder (Fig. 2).

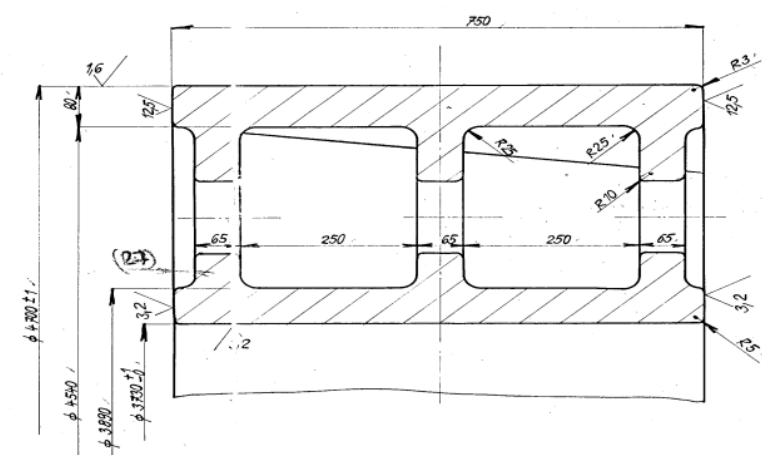

Figure 2: Support ring profile cross section (cut-out from the Drawing)

The object of the measurement shown in Fig. 1 was the support ring, on which surface cracks began to appear. The paper mill ordered a new support ring. Its delivery date was 2 months. During this time, the cracks represented a major risk of the machine failure, which could result in leaks of lime and hydroxide to the environment. For this reason, cracks were checked several times daily by maintenance personnel. To effectively perform that check which necessitated measurement of the actual length of the cracks identified, it was always necessary to either slow down or stop the machine/line. However, these interventions have had a negative impact on the operation of the production line as a whole, and therefore the demand for urgent creation and deployment of a reliable system for automated crack detection and identification of their immediate lengths has arisen.

To obtain such measurement system as fast as possible, starting measurement was carried out using instrumentation available at the laboratories of the CU FSPE BEL and of the CTU FME Dep. 12110.

We considered to find a suitable robust solution for continuous two-month operation:

a) Use of an ultrasound gauge

b) Use of a linear laser $650 \mathrm{~nm}, 5 \mathrm{~mW}$ illumination

c) Use of the Thorlabs LC100 1D camera (profilemeter)

d) Image analysis (FullHD Camera, Stable LED Lighting)

The application of ultrasound gauge proved to be very problematic in the initial phase because of the dimensions of the support ring and because of the need of a contact between the ultrasound units and the measured surface. Although the projected laser beam showed changes in intensity at the cracks edges, the resulting sensitivity and accuracy of this measurement was however strongly dependent on the purity of the contact surfaces - when freshly lubricated, it stopped working completely. The recording by the $1 \mathrm{D}$ camera showed a lack of sensitivity and, moreover, was negatively affected by impurities on the measured surface, which could not be distinguished from the crack records. For those reasons, the final detection system was designed, based on the image analysis.

\section{Method}

The final measuring system was designed on the basis of a testing measurement using Dewetron equipment for measuring and controlling light conditions for determining the necessary parameters of lighting units and sensitive full HD camera with a wide set-up possibilities in terms of exposure conditions, sampling frequency and other parameters affecting the contrast and brightness of the pictures taken. In order to protect the final apparatus as much as possible from the heat and other environmental influences, it was placed under the support ring between two support rollers in the cover boxes (Fig. 3).

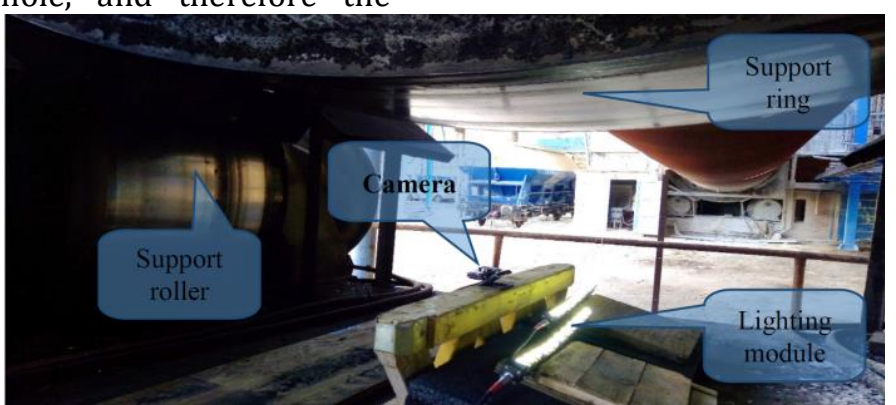

Figure 3: Measurement spot installation under preliminary testing 
The described testing measurement was supplemented by check of the geometric accuracy of the support ring and the supporting rollers using the Somet ČSN 251811 analogue device (ranged $10 \mathrm{~mm}$, accuracy +/- $0.01 \mathrm{~mm}$ ).

Fig. 4 shows the final installation that was made up of two boxes. The camera and lighting modules lamps were placed in the first box under the measured support ring. In the second box, the control electronics was placed - a PC with the acquisition program created in Matlab 2017 environment and a control unit for starting and termination the measurements based on the Arduino Uno platform. The system has recorded one revolution of the cylinder each two hours. The measurement was started and stopped by a trigger signal from a conventional optical bar located on the side of the measuring box. The protective boxes were wooden with lamellar floor to allow air flow-through for sufficient cooling of their inner space. For the same purpose, the box with the control electronics was equipped with a lamellar roof.

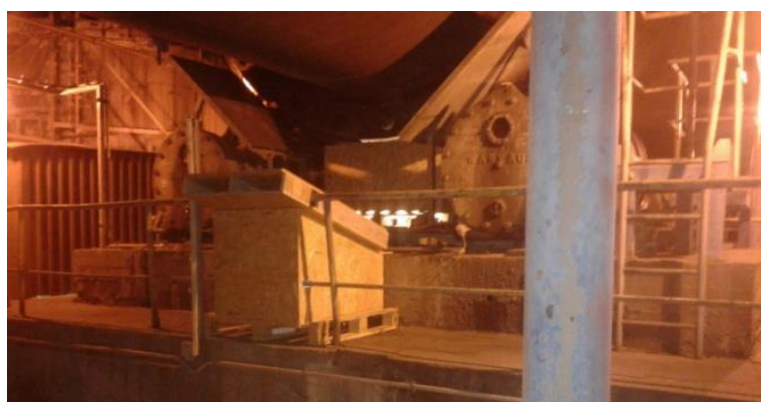

Figure 4: Final measurement system installation

\section{Data Processing}

One revolution provided 1650 gray scale images. In order to reduce the data exchange between the control PC, cloud storage and the PC for final data processing in the control room, the reduced field of view sized of 1918 × 370 px was applied on saved images as raw data (Figure $5 \mathrm{a}$ ).

During saving process, the images have been processed by application of smoothening filter in horizontal direction to highlight horizontally oriented objects - because the cracks occurred in a direction parallel to the axis of the mixing cylinder only.
By this way, many artifacts were disposed. After that, the mathematical morphology algorithms have been applied all images were binarized by thresholding using the Otsu's method (Figure 5b).

The binary images were processed for automatic detection of cracks and their lengths evaluation. Assessed data were sent to the cloud storage from where the authorized person could download it to a computer in the control room where they could be further processed.

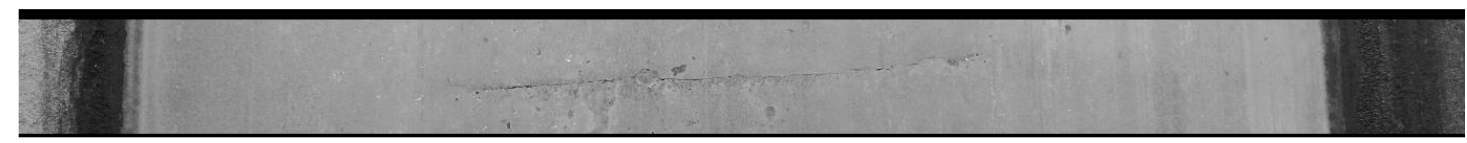

(a)

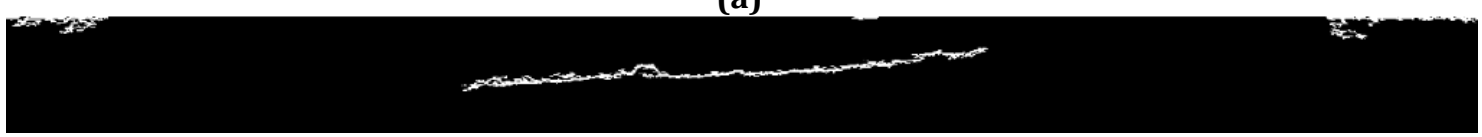

(b)

Figure 5: (a) Raw image taken by a camera, (b) Highlighted crack after the image processing

The images prepared according to Fig. 5a were used to manually evaluate the length of the identified cracks by the authorized personnel. This evaluation was based on the output of the above mentioned automatic detection of the cracks, which identified images with suspicion of a crack. The reason for this procedure was to verify reliability and sensitivity of the automatic identification of the cracks and their lengths.
The number of present cracks and two hours monitoring frequency allows to perform this manual verification one time in a working shift.

The automatic detection algorithm was based on "regionprobs" Matlab function for image processing [1]. It examines white objects in matrix of processed binary image in terms of their area and eccentricity.

A found object was identified as a crack if it has minimum area of white and its eccentricity was close to one. 
The algorithm was extended by supplementary confirming condition requiring the repeating of the result in certain number of consecutive images. This number was determined by rotation speed of the bearing ring.

\section{Results}

The contact measurement of the geometry shape of the contact surfaces of the support ring and the support rollers showed strong wobbling (Fig. 6). The measurements were made at a distance of $8-10 \mathrm{~cm}$ from both ring faces.

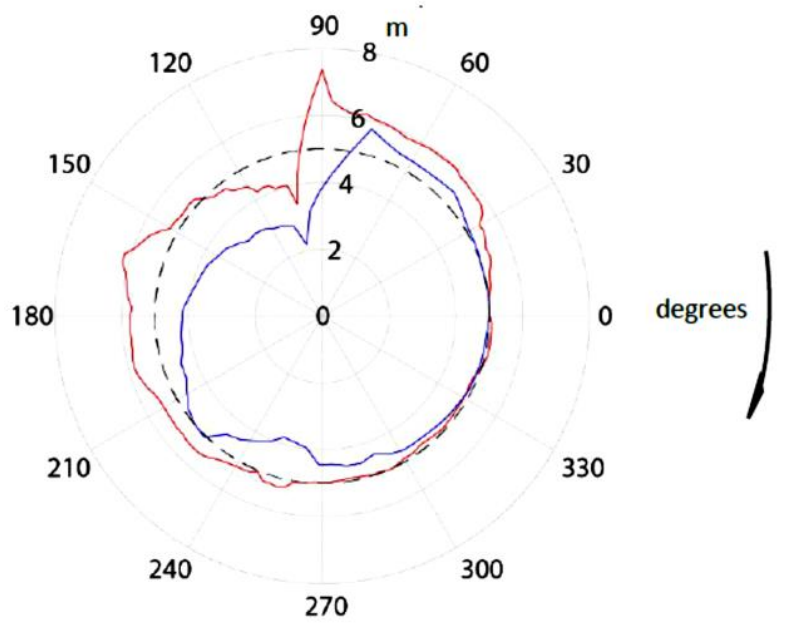

Figure 6: Measured shape characteristics of the support ring rolling surface (red - end close to the higher face in direction of the rake angle of the mixing cylinder; blue - end close to the lower face; the scale for identified shape deviations: 1000:1)

The maximum change of the actual ring diameter was determined to $3.6 \mathrm{~mm}$ by repeated reading.

This is $2.1 \mathrm{~mm}$ more than the drawing radius tolerance (Fig. 2). A step-like pattern of the measured surface curve caused induction of huge load to the bearing ring due to inertial effect of massive mill tube. The position of cracks identified by automated algorithm were localized close to the highly deformed area of the ring surface. Ovality of the support rollers has been found in the order of max. tenths of $\mathrm{mm}$ without any step-wise changes.

Fig. 7 presents a user interface on a computer in the control room, which was at disposal to an authorized worker of the paper mill.

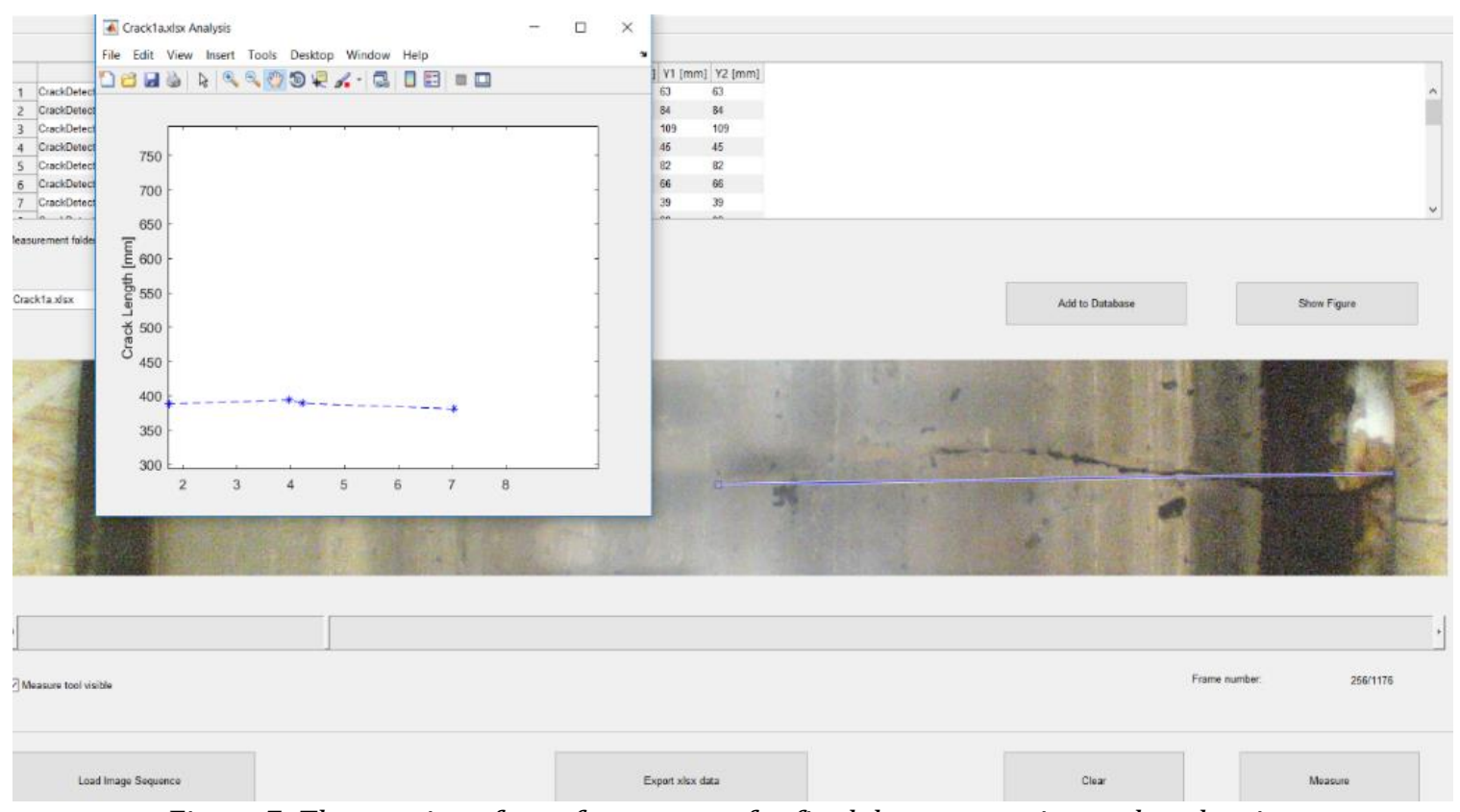

Figure 7: The user interface of a program for final data processing and evaluation 
A graphical user interface, shown in the Fig. 7, enables image upload from a cloud storage. The indicated images with suspicion of a crack were measured with a visible measuring tool. The evaluation interface contains a database in the top of the screen where the cracks are sorted according its individual measured lengths.
The crack length development as a function of recorded time is generated automatically from values stored in the database in a floating window. An example of the outputs for the three largest cracks is shown in Fig. 8.
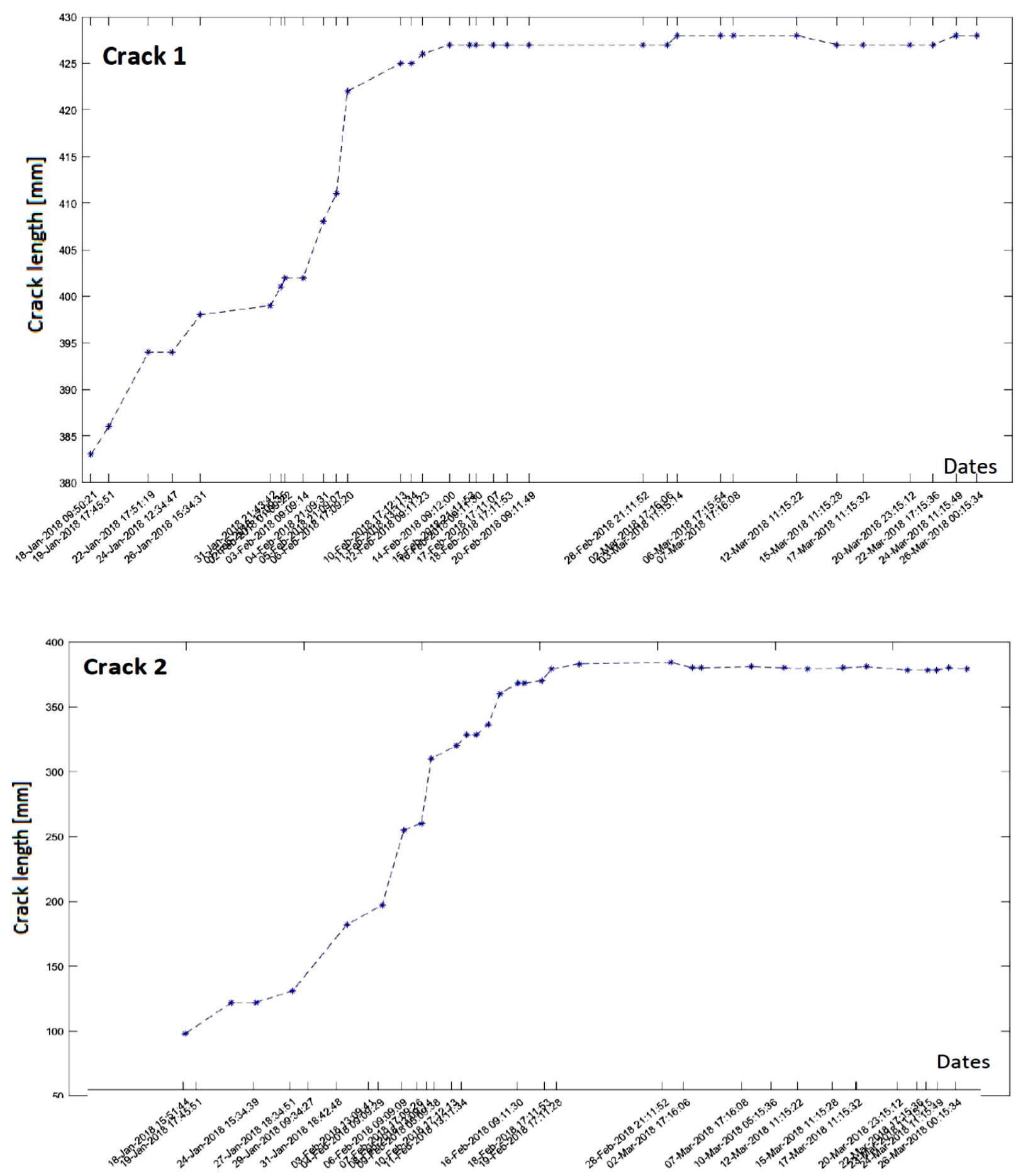


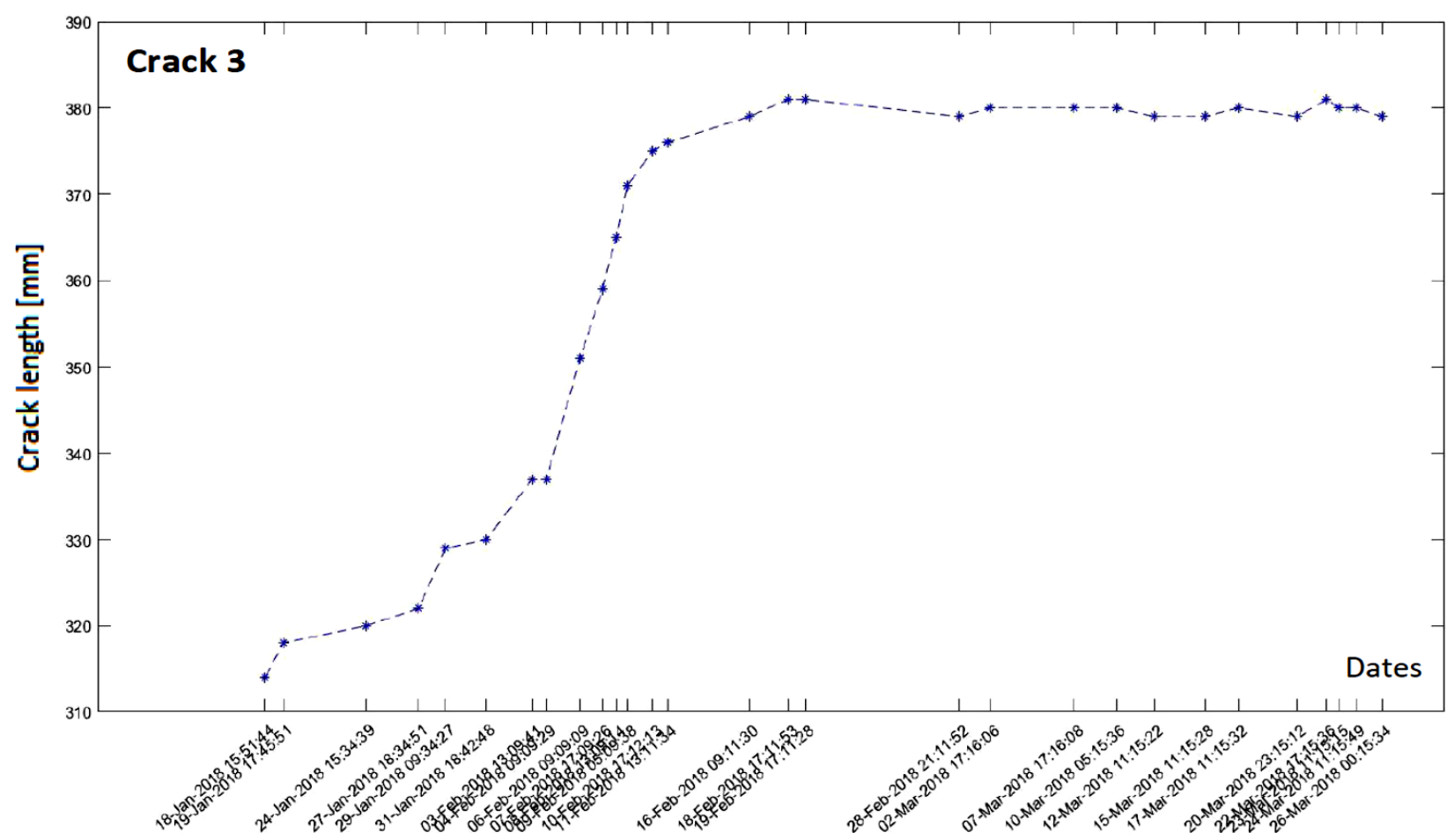

Figure 8: Output of the system - temporal cracks length development

\section{Conclusions}

The aim of the paper is to highlight the importance and possibilities of continuous monitoring of the state of machine components which enables effective service operations planning. Besides that, it also facilitates service interventions because the reason of the problem is identified. Thus, the service intervention can be very effective. In early service intervention, larger damages are avoided and both service costs and its technological difficulty is also reduced. Last but not least, the on-going monitoring brings valuable data for designing and dimensioning of the monitored components.

Here, the application of the described semiautomatic system for detecting damages of the machine component eliminated the need of frequent shutdowns of the machine which was previously necessary for cracks length measurement. Online follow-up of the cracks length development further enabled quite accurate advanced estimation of the time for temporary repair of the support ring by weld rectifications of the cracks and reinforcements of the adjacent ring faces by metal plates welded in the critical places.

Surprisingly enough, pumping of about half of the cylinder content in the preparation for long-term shutdown of the production line resulted in stopping of further growth of cracks lengths, as can be seen in right parts of presented graphs.
Thus, with regard to the remaining days to the exchange of the support ring, the planned temporary welding repairs could be cancelled.

Another interesting finding was that some cracks identified on the support ring in one measurement were not found in subsequent ones. This crack healing is probably attributable to the effect of rolling between the ring and support rollers [2].

It implies that manual or visual surface examination itself does not provide complete information without database of actual and former crack lengths development. For extent the system as a predictive maintenance tool, it would be beneficial to amend it by means for analyses of subsurface layers.

\section{Acknowledgements}

(Facultative Field). This work was supported by project number SGS17/176/OHK2/3T/12.

\section{References}

[1] Mathworks web pages. Available from: https://www.mathworks.com/help/images/ref/ regionprops.html

[2] Gao H, Ai Z, Yu H, Wu H, Liu X. Analysis of Internal Crack Healing Mechanism under Rolling Deformation. Kuzyk MG, ed. PLoS ONE. 2014;9(7):e101907. 